\title{
ASPECTOS ÉTICOS DE LOS ESTUDIOS DE BIODISPONIBILIDAD Y BIOEQUIVALENCIA DE PRODUCTOS FARMACÉUTICOS CONTENIDOS EN LAS LEGISLACIONES DE AMÉRICA LATINA*
}

\begin{abstract}
Resumen: El objetivo de este artículo es identificar los criterios éticos establecidos en algunos países con normatividad vigente en biodisponibilidad/bioequivalencia y determinar si tales criterios protegen adecuadamente a los sujetos que participan en estos estudios. Para recabar la información se realizó una encuesta vía e-mail a los responsables en materia de biodisponibilidad/bioequivalencia de las agencias reguladoras de medicamentos de Argentina, Bolivia, Brasil, Chile, Colombia, Costa Rica, Ecuador, México, Paraguay, Perú, Uruguay y Venezuela. Las conclusiones apuntan a que las normativas vigentes recogen de manera desigual los aspectos éticos necesarios para proteger a los participantes en estos estudios; uniformar las exigencias éticas permitirá desarrollar de manera más rápida la legislación en aquellos países que aún no la tienen y consolidar los esfuerzos de armonización regulatoria de los productos farmacéuticos en la Región.
\end{abstract}

Palabras clave: Ética, biodisponibilidad, bioequivalencia, legislación

\section{INVESTIGATIONS' ETHICAL ASPECTS REGARDING PHARMACEUTICAL PRODUCTS' BIODISPONIBILITY AND BIOEQUIVALENCE IN LATIN AMERICAN LEGISLATIONS}

\begin{abstract}
This paper pretends to identify the ethical criteria established in certain countries with up-to-date norms on biodisponibility/bioequivalence, and to determine if such criteria really protect participants in these investigations. In order to obtain information a questionnaire on biodisponibility/bioequivalence topics was sent via e-mail to the medicaments' regulating agencies in Argentina, Bolivia, Brasil, Chile, Colombia, Costa Rica, Ecuador, México, Paraguay, Perú, Uruguay and Venezuela. Conclusions show that up-to-date norms regarding necessary ethical aspects to protect people who participate in those investigations are unequal Ethical requirements should be uniformed so as to allow a quicker legislation development in those countries that still lack it. This would also strengthen the region's pharmacaeutical regulations
\end{abstract}

Key words: Ethics, biodisponibility, bioequivalence, legislation

\section{ASPECTOS ÉTICOS DOS ESTUDOS DE BIODISPONIBILIDADE E BIOEQUIVALÊNCIA DE PRODUTOS FARMACÊUTICOS CONSTANTES NAS LEGISLAÇÕES DA AMÉRICA LATINA}

Resumo: O objetivo deste artigo é identificar os critérios éticos estabelecidos em alguns países com normatividade vigente em biodisponibilidade / bioequivalência e determinar se tais critérios protegem adequadamente os sujeitos que participam desses estudos. Para obter a informação foi realizada uma pesquisa via e-mail com os responsáveis em matéria de biodisponibilidade / bioequivalência das agências reguladoras de medicamentos da Argentina, Bolívia, Brasil, Chile, Colômbia, Costa Rica, Equador, México, Paraguai, Peru, Uruguai e Venezuela. As conclusões apontam que as normativas vigentes recolhem de forma desigual os aspectos éticos necessários para proteger os participantes nestes estudos; uniformizar as exigências éticas permitirá desenvolver de forma mais rápida a legislação em aqueles países que ainda não a possuem e consolidar os esforços de harmonização regulatória dos produtos farmacêuticos na região.

Palavras-chave: Ética, biodisponibilidade, bioequivalência, legislação

\footnotetext{
* Fogarty NIH Research Grant \#D43 TW 06056

** Área de Investigación, Centro Nacional de Control de Calidad, Instituto Nacional de Salud

Correspondencia: lemoreno70@hotmail.com
} 


\section{Introducción}

"La ética promueve una investigación científica de calidad intrínseca, no guiada por lo promocional o por el egoísta engrosamiento del listado de publicaciones personales. La investigación científica se guía fundamentalmente por dos grandes principios: la búsqueda de la verdad y el recto servicio a la sociedad, con una clara perspectiva de respeto por las personas(1)". Desde este punto de vista, los estudios de biodisponibilidad (BD) y bioequivalencia (BE) tienen un objetivo ético al ser herramientas diseñadas para garantizar la calidad y seguridad del medicamento permitiéndole cumplir su rol social: beneficiar a la persona a la cual le es administrado. Dentro de los principales valores humanos relacionados con el medicamento tenemos: "La solidaridad, que debe manifestarse en la voluntad de poner el medicamento al servicio de todos los hombres, y la verdad, como guía de la bondad y eficacia del medicamento( $1, p .103)$ ".

Las actividades de la Organización Mundial de la Salud (OMS) relativas a los medicamentos se basan, principalmente, en el documento "WHO medicines strategy: framework for action in essential drugs and medicines policy 2002-2003". El objetivo de la estrategia es aprovechar al máximo los medicamentos esenciales para salvar vidas y mejorar el estado de salud de la población. Uno de los cuatro objetivos de esta estrategia consiste en aumentar la calidad y seguridad de los medicamentos(2).

La biodisponibilidad es un tipo de estudio que se realiza en humanos y que permite determinar la velocidad de absorción y la cantidad de un principio activo en la circulación sistémica o en la orina después de la administración de un medicamento. La bioequivalencia es un estudio de biodisponibilidad comparativo entre un producto farmacéutico en estudio y uno de referencia.
Cuando se administra un medicamento genérico, al cual se le ha realizado un estudio de bioequivalencia, y demuestra que alcanza la misma velocidad y concentración de principio activo que el medicamento innovador, se está asegurando la misma respuesta terapéutica en el paciente que la que se obtendría con el medicamento innovador, logrando de esta manera cumplir con uno de los cuatro objetivos de la OMS en materia de medicamentos: poner al alcance de la población medicamentos genéricos que tengan un costo mucho menor que aquellos innovadores y, a la vez, que satisfagan las exigencias de calidad y eficacia(3).

Un estudio de bioequivalencia típico consiste en administrar a voluntarios dos medicamentos-el de estudio y el de referencia-en dos tiempos separados por un período denominado de lavado, para asegurar que el primer medicamento es eliminado y no interfiere con el segundo. Antes y después de comenzar con la administración de los medicamentos, y durante períodos definidos, se toman muestras de sangre y/o orina (Etapa Clínica) que son analizadas en el laboratorio para determinar su concentración. El aumento y la caída de las concentraciones en el tiempo, en cada sujeto del estudio, proveen la información acerca de cuánto principio activo del medicamento de prueba y de referencia es absorbido por el cuerpo (Etapa Analítica). Con los datos obtenidos se construyen curvas de concentración/ tiempo y se calcula el área bajo la curva (AUC); éstas se elaboran para cada sujeto del estudio y los valores son calculados estadísticamente (Etapa Estadística)(4).

Es importante señalar que no todos los medicamentos necesitan un ensayo de bioequivalencia. Básicamente se realizan estos estudios a los siguientes productos: tabletas orales (de liberación inmediata o modificada) que están indicadas para condiciones graves (antibióticos, antivirales, cardiovasculares, entre otros); aque- 
llos con estrecho margen terapéutico, es decir, que tienen la concentración terapéutica y tóxica muy cercanas; los medicamentos con absorción incompleta, baja solubilidad, inestabilidad; y aquellos con evidencia de problemas con la biodisponibilidad. Los demás medicamentos demuestran su equivalencia terapéutica a través de pruebas in vitro, estudios farmacocinéticos, farmacodinámicos y estudios clínicos comparativos ${ }^{1}$.

En América Latina se presentan tres tipos de medicamentos: los innovadores, los no innovadores con denominación de marca y los genéricos. La calidad de los no innovadores con denominación de marca y de los genéricos se determina a través de su equivalencia farmacéutica y terapéutica con respecto al producto innovador. La equivalencia farmacéutica se demuestra verificando que el fabricante implemente, adecuadamente, las Buenas Prácticas de Manufactura (BPM) y las Buenas Prácticas de Control de Calidad (BPCC) en su laboratorio; que el producto cumpla con las especificaciones de controles fisicoquímicos exigidos para el producto en la farmacopea y/o textos oficiales adoptados en el país; y que las características relativas a la forma de dosificación, dosis e indicaciones de uso rotuladas sean equivalentes a las del innovador. La equivalencia terapéutica se determina mediante los estudios de bioequivalencia. La demostración de equivalencia farmacéutica es ya un requisito establecido en las reglamentaciones para los registros sanitarios en los países de la región; sin embargo, aunque se tiene plena conciencia de la importancia y urgencia de cumplir con el requerimiento de la demostración de equivalencia te-

Departamento de Salud y Servicios Humanos de los Estados Unidos. Administración de Alimentos y Medicamentos (FDA). Centro de Evaluación e Investigación de Fármacos (CDER). Estudios de biodisponibilidad y bioequivalencia para productos farmacéuticos administrados oralmente. Consideraciones generales. Octubre 2000. [Sitio en Internet] Disponible en: http://www.fda.gov/cder/guidance/index.htm rapéutica, en la mayoría de los países aún no existen normativas de bioequivalencia.

La implementación de normativas de biodisponibilidad/bioequivalencia en todos los países de la región es ya inaplazable, debido al papel fundamental que cumplen, desde el punto de vista regulativo, para garantizar la eficacia y seguridad de todos los medicamentos comercializados. El presente trabajo pretende actualizar la información referente a los países que tienen legislación sobre biodisponibilidad/ bioequivalencia y recoger de las normativas los aspectos éticos necesarios que deben guiar el desarrollo de estos estudios.

\section{Materiales y métodos}

Con la información disponible de la Reunión de Expertos en Bioequivalencia de Productos Farmacéuticos, OPS/OMS, Caracas, Venezuela (13-15 Enero 1999)(5), donde participó la mayoría de países del Mercado Común del Sur (MERCOSUR), de la Comunidad Andina y de Centroamérica, se procedió a clasificar a los países de la región de la manera como se indica en la Tabla $\mathrm{N}^{\mathrm{o}} 1$.

Se realizó una encuesta vía correo electrónico a los responsables en materia de biodisponibilidad/bioequivalencia de las agencias reguladoras de medicamentos de los países señalados para actualizar la información de la Tabla $N^{o} 1$ hasta enero de 2004. Se les propuso el siguiente cuestionario:

- En su país, la regulación para el registro de medicamentos, ¿exige o exigirá en el futuro pruebas de biodisponibilidad / bioequivalencia?

- Las legislaciones de biodisponibilidad/bioequivalencia en su país, ¿incluyen o hacen referencia a alguna Declaración Internacional, Principio o Código de Ética? 
Aspectos Éticos de los Estudios de Biodisponibilidad y Bioequivalencia - L. Moreno

Tabla N ${ }^{0}$ 1. Legislación sobre biodisponibilidad/bioequivalencia en algunos países de América Latina, enero 1999.

\begin{tabular}{|c|c|c|c|c|}
\hline $\begin{array}{l}\text { País/ } \\
\text { Legislación }\end{array}$ & $\begin{array}{l}\text { Grupo } 1 \\
\text { Legislación } \\
\text { vigente }\end{array}$ & $\begin{array}{c}\text { Grupo } 2 \\
\text { Legislación, } \\
\text { s/ aplicación }\end{array}$ & $\begin{array}{c}\text { Grupo } 3 \\
\text { Proyectos } \\
\text { de Ley /Estudios }\end{array}$ & $\begin{array}{c}\text { Grupo } 4 \\
\text { S/ información } \\
\text { y/o avances }\end{array}$ \\
\hline Argentina & ANMAT 3185/99 & & & \\
\hline Bolivia & & & & $\mathrm{X}$ \\
\hline Brasil & ANVISA $9787 / 99$ & & & \\
\hline Chile & & & & $\mathrm{X}$ \\
\hline Colombia & & Decreto $677 / 95$ & & \\
\hline Costa Rica & & Reg. 99 & & \\
\hline Ecuador & & & & $\mathrm{X}$ \\
\hline México & NOM 177-SSA-1-98 & & & \\
\hline Paraguay & & & & $\mathrm{X}$ \\
\hline Perú & & & & $\mathrm{X}$ \\
\hline Uruguay & & & & $\mathrm{X}$ \\
\hline Venezuela & & & Norma BD /BE 98 & \\
\hline
\end{tabular}

- ¿Qué instituciones realizan estudios de biodisponibilidad/bioequivalencia en su país?

- ¿Cuál es el costo promedio y tiempo aproximado para realizar un estudio de biodisponibilidad/bioequivalencia en su país?

- ¿Cuáles, cree usted, que son las perspectivas de los estudios de biodisponibilidad/bioequivalencia en su país?

- ¿Es usted optimista o pesimista?

- ¿Dónde estarán en 5-10 años?

En seguida, las legislaciones fueron evaluadas y clasificadas en relación con las orientaciones éticas de las investigaciones en seres humanos(6). Primero, se determinó si el contenido de las legislaciones incluye o hace referencia a:

- Comité o Comisión de Ética.

- Declaración de Helsinki VI / Pautas CIOMS 2002.

- Consentimiento del voluntario y/o paciente.

- Principios y Códigos Nacionales de Ética.

- Sin orientación ética.
Además, se determinó si la legislación explicitaba los siguientes aspectos:

- Obligaciones de los investigadores.

- Derecho a tratamiento y compensación para sujetos perjudicados.

- Incentivos para participar en el estudio.

- Beneficios y riesgos.

- Selección de voluntarios.

- Características del protocolo por presentar.

- Protección de la confidencialidad.

- Conflicto de intereses.

\section{Resultados}

La situación actual es la siguiente (ver Tabla 2):

- Grupo 1: en estos países se realizan ensayos de $\mathrm{BD} / \mathrm{BE}$ según la legislación vigente. Es importante señalar que la evolución de la legislación, la acreditación de centros autorizados para realizar estos estudios y la publicación de documentos complementarios 
(por ejemplo, el Manual de Buena Práctica en Biodisponibilidad y Bioequivalencia CIBIO-Brasil) han permitido estandarizar los procedimientos y mejorar su calidad. En estos países, el costo promedio fluctúa entre treinta y cien mil dólares, y el tiempo promedio entre ocho y cuarenta semanas, siendo buena la perspectiva de aumento en el número de los estudios.

- Grupo 2: todos estos países cuentan con leyes que regulan los estudios, además de ser requisito para la inscripción, control y registro de medicamentos; sin embargo, falta todavía la reglamentación para hacer efectiva la ley.

- Grupo 3: estos países cuentan, a la fecha, con proyectos de ley y/o se encuentran en la etapa de preparación de normatividad para incorporar este tema en sus legislaciones de productos farmacéuticos. No obstante, las perspectivas para poner en marcha estos estudios son a mediano plazo ( 5 a 8 años).

- Grupo 4, la situación no ha cambiado mucho desde enero de 1999 a la fecha.

Tabla No 2. Legislación sobre biodisponibilidad/bioequivalencia en algunos países de América Latina, enero de 2004.

\begin{tabular}{|c|c|c|c|c|}
\hline País/ Legislación & $\begin{array}{l}\text { Grupo } 1 \\
\text { Legislación } \\
\text { vigente }\end{array}$ & $\begin{array}{c}\text { Grupo } 2 \\
\text { Legislación, } \\
\text { s/ aplicación }\end{array}$ & $\begin{array}{c}\text { Grupo } 3 \\
\text { Proyectos } \\
\text { de Ley /Estudios }\end{array}$ & $\begin{array}{c}\text { Grupo } 4 \\
\text { S/ información } \\
\text { y/o avances }\end{array}$ \\
\hline Argentina & ANMAT 3185/99 & & & \\
\hline Bolivia & & & & $X$ \\
\hline Brasil & ANVISA 9787/99 & & & \\
\hline Chile & & & Proyecto Norma & \\
\hline Colombia & & $\begin{array}{c}\text { Decreto } 677 / 95 \\
\text { Res. } 1400 / 2001 \\
1890 / 2001\end{array}$ & & \\
\hline Costa Rica & & Reg. 28466-S & & \\
\hline Ecuador & & & & $\mathrm{X}$ \\
\hline México & NOM 177-SSA-1-98 & & & \\
\hline Paraguay & & & & $X$ \\
\hline Perú & & & Norma en elaboración & \\
\hline Uruguay & & & & $\mathrm{X}$ \\
\hline Venezuela & & Ley 37.006 & & \\
\hline
\end{tabular}

Los resultados del análisis de las orientaciones éticas se resumen en la Tabla $\mathrm{N}^{\mathrm{o}} 3$. A continuación se presenta la situación de cada país.

\section{Argentina}

E1 25 de junio de 1999, la ANMAT (Administración Nacional de Medicamentos, Alimentos y Tecnología Médica) promulgó la Disposición 3185/99 "Requerimiento de Estudios de Bioequivalencia", contemplando comenzar con un listado de drogas críticas (15 en total) seleccionadas teniendo en cuenta dos aspectos: 1) Riesgo Sanitario Alto, es decir, probabilidad de que aparezcan complicaciones de la enferme- dad, amenazantes para la vida o para la integridad psicofísica de la persona, y/o de reacciones adversas graves, cuando la ventana terapéutica del medicamento sea estrecha, y 2) drogas a las que se les exige ensayos de bioequivalencia en Alemania, EE.UU. y Canadá(7).

Los requerimientos éticos de la Disposición 3185/99 hacen referencia a la Disposición 5330/ 97 en cuyo capítulo XI se indica:

"Comité de Ética: Los investigadores principales deberán garantizar la participación de un Comité de Ética, independiente de los investigadores intervinientes en el ensayo clíni- 
Tabla N ${ }^{\circ}$ 3. Orientaciones éticas en las normativas sobre biodisponibilidad/bioequivalencia en algunos países de América Latina

\begin{tabular}{|c|c|c|c|c|c|}
\hline País & Comité de Ética & $\begin{array}{c}\text { Declaración } \\
\text { Helsinki/Pautas } \\
\text { CIOMS }\end{array}$ & $\begin{array}{c}\text { Consentimiento } \\
\text { del voluntario } \\
\text { y/o paciente }\end{array}$ & $\begin{array}{l}\text { Códigos } \\
\text { Nacionales } \\
\text { de Ética }\end{array}$ & $\begin{array}{l}\text { Sin orientación } \\
\text { ética }\end{array}$ \\
\hline Argentina & $X$ & & $\mathrm{X}$ & & \\
\hline Brasil & $X$ & & $\mathrm{X}$ & & \\
\hline México & $X$ & $\mathrm{X}$ & $\mathrm{X}$ & & \\
\hline Colombia & & & & & $\mathrm{X}$ \\
\hline Costa Rica & & & $\mathrm{X}$ & $\mathrm{X}$ & \\
\hline Venezuela & & $X$ & $\mathrm{X}$ & & \\
\hline
\end{tabular}

co, que estará compuesto por personas provenientes de diferentes ámbitos. Esta administración promoverá la formación de tales Comités en diversos puntos del país.

Consentimiento Informado: Será requisito indispensable para la autorización de un ensayo clínico la presentación de un formulario de consentimiento informado, que será firmado por el paciente en presencia de, por lo menos, un testigo, y que sólo será válido cuando exista constancia fehaciente de que el paciente ha sido informado de la confidencialidad de la información, de los objetivos, métodos, ventajas previstas, alternativas terapéuticas y posibles riesgos inherentes al estudio, así como de las incomodidades que pueda acarrearle, y de que es libre de retirar su consentimiento de participación en cualquier momento, sin explicar las causas. Ello no deberá derivar en perjuicio alguno para el paciente/voluntario sano. Asimismo, deberá constar que el patrocinante y/o investigador proveerán en forma gratuita la medicación en estudio".

\section{Brasil}

El Ministerio de Salud de Brasil dictó la Ley $\mathrm{N}^{0} 9.787$ (10 febrero 1999), disponiendo la vigilancia sanitaria a la que estarán sujetos los medicamentos genéricos. En concordancia con esta ley, la ANVISA (Agencia Nacional de Vigilancia Sanitaria) aprobó el Reglamento Técnico para Medicamentos Genéricos mediante Resolución
$\mathrm{N}^{0}$ 391, del 9 agosto de 1999, donde se indica que "...las pruebas de biodisponibilidad en general y las pruebas de bioequivalencia, como verificación de la equivalencia terapéutica, el registro y la intercambiabilidad de los medicamentos genéricos, se regirán por el presente reglamento ${ }^{2 "}$. En el anexo VI de esta resolución se publicó una primera lista de 100 medicamentos de referencia para realizar estas pruebas.

En lo referente a los aspectos éticos contenidos en la Resolución No 391, se menciona en el ítem 5, Criterios para Pruebas de Bioequivalencia de Medicamentos Genéricos, 5.1. Etapa Clínica: "h) el número mínimo de voluntarios sanos, generalmente 24 , con edades entre $18 \mathrm{y}$ 50 años deberán ser capaces de dar su consentimiento libre e informado [...]; o) el Proyecto de Investigación, Protocolo Experimental y el Consentimiento Libre e Informado deberán ser sometidos a un Comité de Ética de la Investigación (CEP) acreditado por el Comité Nacional de Ética e Investigación (CONEP) del Consejo Nacional de Salud (MS); p) los voluntarios participantes en estudios clínicos, que necesiten confinación, deberán permanecer en un lugar apropiado que atienda las Buenas Prácticas Clínicas (BPC), bajo responsabilidad de un médico".

Agencia de Vigilancia Sanitaria de Brasil. Reglamento Técnico para Medicamentos Genéricos - Resolución 391. Revisado el 17-09-03. [Sitio en Internet] Disponible en: http://www.anvisa.com 


\section{México}

La Norma Oficial Mexicana, NOM-177SSAI- $1998^{3}$, establece las pruebas y procedimientos para demostrar que un medicamento es intercambiable. Los requisitos a los que deben sujetarse los terceros autorizados que realicen las pruebas se basan en lo dispuesto en la Ley General de Salud, en el Reglamento de la Ley General de Salud en Materia de Investigación para la Salud y en las Buenas Prácticas Clínicas.

La Norma Oficial Mexicana NOM-177SSAI-1998, en el ítem 8.2.2., señala: "Cada protocolo debe ser revisado y aprobado por el coordinador general o investigador principal, ser sometido a los comités de ética y de investigación de la institución responsable del estudio". En 8.3.6. se indica: "En caso de existir una relación de subordinación entre los voluntarios y los investigadores se debe atender a lo dispuesto en el Reglamento de la Ley General de Salud en Materia de Investigación para la Salud y demás disposiciones aplicables". Respecto al consentimiento informado, el ítem 8.3.7 menciona: "...los voluntarios deben firmar una carta de aceptación para participar en el estudio, carta de consentimiento informado y demás requisitos del Reglamento de la Ley General de Salud en Materia de Investigación para la Salud". En 8.3.9 señala: "Los voluntarios deben ser remunerados en función del riesgo y del tiempo empleado para el estudio".

\section{Colombia}

El Decreto 677, de $1995^{4}$, reglamenta el Régimen de Registros y Licencias, el Control

Secretaría de Salud México. Norma Oficial Mexicana NOM-177-SSA1-1998. Establece las pruebas y procedimientos para demostrar que un medicamento es intercambiable. Revisado el 30-08-03. [Sitio en Internet] Disponible en: http://www.ssa.gob.mx

4 Instituto Nacional de Vigilancia de Medicamentos y Alimentos de Colombia. Normatividad. Revisado el 02-09-03. [Sitio en Internet] Disponible en: http://www.invima.gov.co de Calidad y la Vigilancia Sanitaria de Medicamentos. En el Artículo 22, inciso ñ, se indica que para la evaluación farmacéutica se requieren: "...resultados de los estudios de biodisponibilidad y bioequivalencia para los productos definidos por el Instituto Nacional de Vigilancia de Medicamentos y Alimentos (INVIMA). Asimismo, la Resolución 1400/2001, en su Artículo 4, señala: “...se exigirán estudios de Bioequivalencia para los medicamentos que se comercializan en Colombia bajo denominación genérica o de marca, cuando el productor interesado solicite la certificación de intercambiabilidad con el innovador en el mercado". De la misma manera, en el Artículo 5, se indica que: "...los grupos farmacológicos que deben presentar estudios de Bioequivalencia in vivo son: a) Anticonvulsivantes: b) Inmunosupresores; c) Medicamentos definidos por el INVIMA cuando lo considere pertinente por sus características de alto riesgo, tales como toxicidad, margen terapéutico estrecho y comportamiento farmacocinético, previo concepto y sustentación científica de la Comisión Revisora, Sala Especializada de Medicamentos".

\section{Costa Rica}

El 29 de febrero de 2000 se publicó el Reglamento de Inscripción, Control, Importación y Publicidad de Medicamentos N ${ }^{0} 28466-S^{5}$, teniendo como sustento jurídico la Constitución Política, La Ley General de la Administración Pública y la Ley General de Salud No 5395, en cuyo texto se mencionan algunos aspectos referidos a la investigación: “Artículo 66.- La investigación experimental clínica en pacientes deberá sujetarse a las normas del Código de Moral Médica [...] Artículo 68.- Ningún profesional podrá someter a una persona a experi-

Ministerio de Salud de Costa Rica. Reglamento de Control Estatal de Medicamentos. Revisado el 05-09-03. [Sitio en Internet] Disponible en: http://www.ministeriodesalud.go.cr/ dirregis/Registro\%20de\%20Medicamentos.htm 
mentación clínica con fines científicos sin que haya antecedentes acumulados por experiencias previas con animales y sin que el sujeto otorgue previamente su consentimiento".

En el artículo 29 del Reglamento $\mathrm{N}^{\circ} 28466-\mathrm{S}$ se indica que "...para la inscripción de productos farmacéuticos multiorigen de riesgo sanitario, deben presentarse estudios de equivalencia terapéutica". El 5 de junio de 2002 se publicó una disposición en la cual se señala que el Consejo Técnico de Inscripción de Medicamentos del Ministerio de Salud exigiría la bioequivalencia a partir de la aprobación del Reglamento para los Estudios de Equivalencia Terapéutica, con la guía correspondiente, el cual está en proceso de elaboración. Se está iniciando con un grupo de siete principios activos los que, a partir de la publicación del Reglamento correspondiente, deberán presentar los estudios de equivalencia terapéutica para obtener o renovar el registro sanitario. En el futuro, se planea ampliar la lista agregando siete principios activos cada año, de forma que en 10 años estarían cubiertos todos los medicamentos asociados con 70 ingredientes activos.

\section{Venezuela}

En La Gaceta Oficial № 37.006, del 3 agosto de 2000, la Comisión Nacional decreta la Ley de Medicamentos ${ }^{6}$. En el artículo 42 se establecen las bases para la realización de los ensayos de bioequivalencia: "El Formulario Terapéutico Nacional, además, deberá contener una lista de medicamentos bioequivalentes, enumerados a partir de sus denominaciones genéricas, con indicación de las formas farmacéuticas, los nombres utilizados en su rotulación, sean éstos de marca o no, y que, de acuer-

Ministerio de Salud República Bolivariana de Venezuela. Ley de Medicamentos. Revisado el 05-09-03. [Sitio en Internet] Disponible en: http://www.venezuelasaludable.gov.ve/ secciones/doc_tecnica/LeyMedicamentos.pdf do con todos los análisis realizados por el Instituto Nacional de Higiene 'Rafael Rangel' o por cualquiera otra institución pública o privada, nacional o extranjera y acreditada por el Ministerio de Salud y Desarrollo Social, sean de igual acción o eficacia terapéutica o susceptibles de ser usados indistintamente, pudiéndose postular como bioequivalentes o de igual eficacia terapéutica cuando lo hayan demostrado según las normas establecidas, disponiéndose de un lapso no menor de tres (3) años para crear la infraestructura y su funcionamiento".

En el Título VI, De los Ensayos Clínicos, los artículos 72 y 73 hacen referencia a algunos aspectos éticos: "Artículo 72. Los ensayos clínicos deberán realizarse en condiciones de respeto a los derechos fundamentales de la persona, y a los postulados éticos que incidan en la investigación biomédica en la que resulten afectados seres humanos siguiendo a estos efectos los contenidos en la declaración de Helsinki sobre Investigación en Humanos y los sucesivos postulados que actualicen la materia.

Artículo 73. Toda persona que participe en estudios de investigación deberá ser previamente informada acerca del alcance y riesgo del ensayo, expresando su consentimiento por escrito y manifestando su pleno conocimiento del tema. Asimismo, deberá ser aprobado por el director del instituto donde se desarrolle la investigación".

\section{Chile}

Existe un proyecto elaborado por el Instituto de Salud Pública denominado: "Norma para realizar estudios de Biodisponibilidad/Bioequivalencia de Productos Farmacéuticos en Chile", encontrándose lista su versión final, así como la lista consensuada de formas farmacéuticas y dosificación (incluye 16 principios activos), el cronograma de aplicación y la lista de productos farmacéuticos comparadores inter- 
nacionales(8). Estos documentos están en espera de su oficialización por el Ministerio de Salud; sin embargo, existe una fuerte presión de los laboratorios y del Colegio Médico en contra de esta norma, que ya hizo fracasar un anterior decreto (el 375) que buscaba implantar la bioequivalencia en Chile(9).

\section{Perú}

Una comisión técnica del Ministerio de Salud, conformada por funcionarios de la Dirección General de Medicamentos, Insumos y Drogas (DIGEMID) y del Centro Nacional de Control de Calidad, está trabajando para elaborar una propuesta de aspectos técnicos y legales. Hubo algunos anteproyectos de ley de medicamentos que contemplaban estos requisitos, pero han quedado estancados. Estos anteproyectos no mencionaban los aspectos éticos. La OPS/ OMS y el Proyecto Vigía, financiado con fondos de la Agencia de los Estados Unidos para el Desarrollo Internacional (USAID), están apoyando técnicamente, desde hace aproximadamente un año, la implementación de la biodisponibilidad/bioequivalencia en Perú.

La legislación vigente en estos países fue contrastada con algunos de los aspectos de ética de la investigación señalados en las Pautas CIOMS 2002(10) y en la Declaración de Helsinki V(11), encontrándose lo siguiente:

- Obligaciones de los investigadores. El investigador tiene la obligación de proteger la vida, salud, privacidad y dignidad de la persona. Se encuentran bien detalladas en la Disposición 5330/97, de Argentina, y en el Reglamento de la Ley General de Salud en Materia de Investigación para la Salud, de México. En ambos casos se contemplan sanciones legales en caso de incumplimiento.

- Derecho a tratamiento y compensación para sujetos perjudicados. El patrocinador tiene la obligación de brindar tratamiento a los participantes que sufran daño como consecuencia de la medicación en estudio. El reporte de las reacciones adversas que presenten los pacientes está contemplado en los reglamentos de Argentina, México y Brasil, mas no el tratamiento y compensación en caso de presentarse.

- Incentivos para participar en el estudio. La Norma Oficial Mexicana, NOM-177-SSAI1998 es la única que señala que los voluntarios deben ser remunerados en función del riesgo y el tiempo empleado para el estudio. Sin embargo, no se precisa que los pagos no debieran ser tan elevados o los servicios médicos tan amplios como para inducir indebidamente a los sujetos a otorgar su consentimiento.

- Beneficios y riesgos de participar en el estudio. Los beneficios en este tipo de estudios son para la sociedad, puesto que participan, en su mayoría, voluntarios sanos, y los riesgos están en función del tipo de medicamento por estudiar. Es necesario precisar en la legislación, además, el monitoreo de la salud de los sujetos durante el estudio.

- Selección de voluntarios (sanos y pacientes), las mujeres como sujetos de investigación. En los casos en que el medicamento sea tóxico se emplearán pacientes en vez de voluntarios. Así está explicitado en el reglamento de Brasil, indicando que esto rige para los medicamentos citotóxicos. La norma de México lo señala para sustancias tóxicas, no especificando cuáles. Ninguna legislación contempla los cuidados que deben tenerse en cuenta, especialmente referidos a evitar el embarazo cuando se incluye mujeres en edad fértil.

- Características del protocolo de investigación por presentar. Debe incluir: identifi- 
cación y calificación del investigador principal y sus colaboradores, lugar donde se realizará el estudio, su objetivo, número de pacientes que se tratarán, criterios de inclusión y exclusión, diseño (cruzado, paralelo, etc.), duración, ensayos y pruebas diagnósticas por realizar previas al estudio, toma de muestras, parámetros que se evaluarán (análisis farmacocinético, parámetros clínicos y bioquímicos), análisis estadístico que se utilizará, y evaluación estadística del tamaño de muestra mínima para que los resultados sean concluyentes.

\section{- Lugar donde se realizarán las pruebas.} Debe ser adecuado para conducir el ensayo de manera eficiente y segura. Las legislaciones de México, Brasil y Argentina señalan que el lugar debe cumplir con las Buenas Prácticas de Laboratorio Clínico. Acerca de las pruebas diagnósticas previas al estudio, la legislación de Argentina se refiere a ellas como pruebas físicas y de laboratorio; la de México señala un listado de exámenes, entre ellos el de VIH (Virus de Inmunodeficiencia Humana); no obstante, no se precisan las consideraciones éticas y de consejería para realizar esta prueba.

- Protección de la confidencialidad. La Disposición 5330/97, de Argentina, y el Reglamento de la Ley General de Salud en Materia de Investigación para la Salud, de México, contemplan los aspectos de confidencialidad y privacidad, respectivamente.

- Conflicto de intereses. Sólo la Norma Oficial de México hace mención al tema.

\section{Discusión}

Se observa un avance en las legislaciones de los diversos países de la región. La inclusión de diversos aspectos relacionados con la ética de la investigación internacional es simi- lar a los estándares exigidos, por ejemplo, en la Guía para Ensayos Clínicos de Productos Farmacéuticos y Biológicos de Estados Uni$\operatorname{dos}(12)$, en la que se recomienda la necesidad de revisión por un Comité de Ética independiente, la información y consentimiento informado del sujeto de investigación, y el respeto por sus derechos, señalados en la Declaración de Helsinki.

El marco legislativo es necesario para implementar y hacer cumplir los diferentes componentes de una política nacional de medicamentos, y para regular las actividades de los diversos actores públicos y privados de este sector. La legislación y regulación farmacéuticas no pueden ser desarrolladas o actualizadas en el vacío, deben estar acordes con el marco legal existente en el país. Es imperativo definir sus objetivos en relación con los objetivos nacionales de salud, las políticas de gobierno y los recursos disponibles.

La legislación debe cubrir los aspectos básicos y permitir la modificación rápida de disposiciones legales de menor nivel que cubran aspectos y detalles técnicos y operacionales.

El consenso político es importante y todos los actores involucrados deben ser consultados.

Existen dos estrategias para efectuar estos estudios: inmediata y gradual. Por la experiencia de los países que han avanzado en el tema es más factible un establecimiento gradual de los requisitos exigidos por la ley. La mayoría de los países que tienen una legislación sobre $\mathrm{BD} / \mathrm{BE}$ han atravesado cuatro etapas: la sustitución de genéricos no es permitida, la sustitución es permitida, la sustitución es promovida, la sustitución con medicamentos genéricos es obligatoria. No es conveniente saltar las etapas, porque se corre peligro de fracasar(13). 
Asimismo, la estrategia gradual debiera comprender:

- La definición acerca de cuáles productos serán sometidos a estudios de bioequivalencia.

- La estandarización de los criterios, procedimientos y técnicas de los tres componentes fundamentales de los estudios de bioequivalencia (clínico, analítico y estadístico).

- La acreditación de laboratorios para realizar estos ensayos.

- La difusión a través de una publicación oficial de los medicamentos analizados.

- Asegurar el cumplimiento de las Buenas Prácticas de Manufactura (GMP).

- El entrenamiento del personal de la agencia regulatoria.

- La aplicación de los dispositivos legales que hagan efectiva su aplicación.

En cuanto a las orientaciones éticas, se observa que son seguidas de desigual manera en la legislación, a diferencia de lo que ocurre en la Comunidad Europea, cuya Directiva sobre Ensayos Clínicos 2001/20/EC señala que todos los ensayos clínicos, incluyendo los estudios de biodisponibilidad y bioequivalencia deben ser diseñados, conducidos y reportados de acuerdo con los principios de buenas prácticas clínicas, siendo obligatorio su cumplimiento para todos los países miembros ${ }^{7}$.

La preocupación de diversos estados latinoamericanos por implementar políticas de biodisponibilidad/bioequivalencia, para asegurar

European Parliament and of the Council. Clinical Trial Directive 2001/20/EC. Official Journal of the European Communities L 121/34. 1.5.2001. [Website] Available at http://medicines.mhra.gov.uk/ourwork/licensingmeds/types/ clintrialdir.htm la calidad de los medicamentos, se enmarca dentro del contexto de una política activa en defensa del derecho a la salud y la eliminación de las inequidades.

Los temas de justicia y equidad en salud pueden ser abordados desde la tradición ética occidental a través de sus dos vertientes:

- Ética deontológica o principialista: dentro de ésta, la que se refiere a la justicia distributiva como bienestar colectivo es la más aceptada. El derecho a la salud es un derecho negativo y el Estado ha de protegerlo; el derecho a la asistencia sanitaria, incluyendo la provisión de medicamentos confiables, es un derecho positivo del cual tiene que hacerse cargo el Estado. La salud no puede ser un problema privado, sino que pasa a ser cuestión pública, política. El primero es una especificación del principio de libertad, en tanto que el segundo se deduce del principio de igualdad(14). Un Estado justo no es sólo el que protege los derechos políticos y civiles de los individuos, sino también el que promueve derechos económicos, sociales, culturales, incluidos los de asistencia sanitaria.

- Ética teleológica: representa el desarrollo sistemático de un tipo de actitud moral que consiste en otorgar mayor importancia a las consecuencias que a los principios; éstos son respetables sólo en la medida en que ello no acarree "malas consecuencias(15)". Aplicado a la justicia sanitaria se refiere a que los administradores y políticos tienen obligación de optimizar la "utilidad pública" de los recursos disponibles, para lo cual han de actuar conforme a los principios y criterios propios de la economía, lo que significa que la justicia distributiva debe regirse siempre por la proporción costo/beneficio; en ese sentido, una pobre performance de medicamentos es no solamente costosa en 
términos financieros, sino también en el número de pacientes que pueden ser afectados por consumir productos inefectivos o de baja calidad(16).

En el detalle de la legislación se muestra que el tratamiento y compensación de sujetos perjudicados, y la consejería y confidencialidad-cuando se realiza el examen de HIV previo al estudio-, entre otros aspectos, no están contemplados en la legislación de aquellos países con normativa. Sería importante que cualquier país que formule una política de medicamentos, e incluya una normativa de bioequivalencia/biodisponibilidad para garantizar su calidad y eficacia, no sólo observe los aspectos técnicos, sino también los éticos.

Finalmente, diremos que el acceso a la salud no se restringe a la atención de las enfermedades, sino que incluye también la prevención, promoción y protección. La satisfacción del derecho a la salud supone un compromiso político basado en los principios éticos de justicia y equidad. El derecho al nivel más alto alcanzable de salud responsabiliza a los gobiernos de la prevención, tratamiento y control de las enfermedades, y de la creación de condiciones que aseguren el acceso a los sistemas de salud, a medicamentos de calidad comprobada y a servicios necesarios para estar sano.

\section{Agradecimientos}

Este trabajo fue posible gracias a una beca Fogarty NIH Research Grant \#D43 TW 06056, que me permitió realizar estudios de postítulo en el Programa Internacional de Investigación Biomédica y Psicosocial en Santiago de Chile.

\section{Referencias}

1. Sapag-Hagar M. El farmacéutico y la ética: al encuentro de una conciencia. Anales de la Academia de Ciencias Farmacéuticas de Chile 1997; 1: 97.

2. Organización Mundial de la Salud. Estrategia farmacéutica de la OMS: Informe sobre los progresos realizados. 56 Asamblea Mundial de la Salud. Marzo 2003

3. Organización Mundial de la Salud. Estrategia revisada en materia de medicamentos. Informe de la Secretaría. 54a Asamblea Mundial de la Salud. Abril 2001.

4. World Health Organization. Quality Assurance of Pharmaceuticals. A compendium of guidelines and related materials. Vol. 1. 1997.

5. Programa de Medicamentos Esenciales y Tecnología. Consulta de Expertos en Bioequivalencia de Productos Farmacéuticos. 13-15 de enero de 1999. Caracas: Organización Panamericana de la Salud. Informe final; Junio 1999.

6. Programa de Subvenciones para la Investigación (PSI). Normas Éticas del PSI para Investigaciones con Sujetos Humanos. Organización Panamericana de la Salud. División de Salud y Desarrollo Humano; 1996.

7. Administración Nacional de Medicamentos, Alimentos y Tecnología Médica de Argentina. Número especial dedicado a biodisponibilidad y bioequivalencia. Boletín para Profesionales 2002; X(34).

8. Milla P. Implicancias de las pruebas de bioequivalencia en el desarrollo de un mercado de medicamentos 
genéricos en paises con un significativo mercado farmacéutico en desarrollo. Brasil: ANVISA/PAHO/ WHO Workshop; Feb. 11-13, 2003.

9. (Redacción). La Peligrosa Fórmula del Gobierno. El Mercurio septiembre 7, 2003; Sección B, Economía y Negocios.

10. Consejo de Organizaciones Internacionales de las Ciencias Médicas. Pautas Éticas Internacionales para la Investigación Biomédica en Seres Humanos. Ginebra: CIOMS; 2002.

11. Asociación Médica Mundial. Declaración de Helsinki. Principios Éticos para la Investigación Médica en Seres Humanos. Escocia: 52a Asamblea General; octubre 2000.

12. Food and Drug Administration. Guideline for Industry Structure and Content of Clinical Study Reports. Center for Drug Evaluation and Research ICH E3; 1996.

13. WHO Policy Perspectives on Medicines. How to develop and implement a national drug policy. $2^{\text {nd }}$ Edition. World Health Organization; 2001.

14. Gracia D. Profesión médica, investigación y justicia sanitaria. En: Gracia D. Estudios de Bioética (Vol. 4). Santafé de Bogotá: El Buho; 1998.

15. Maliandi R. Ética: conceptos y problemas. Buenos Aires: Editorial Biblios; 1991.

16. Videau J-Y. Generic drugs: the hidden issues of quality and cost. Geneve: WHO drug information (Vol. 14); 2000:77. 